\title{
Geant4 Simulation Study of Dose Distribution and Energy Straggling for Proton and Carbon Ion Beams in Water
}

\author{
Qiang Zhao ${ }^{1}$, Zheng Zhang ${ }^{1}$ and Yang $\mathrm{Li}^{1}$ \\ ${ }^{1}$ Beijing Key Laboratory of Passive Safety Technology for Nuclear Energy, School of Nuclear Science and Engineering, North China Electric \\ Power University, Beijing 102206, China
}

\begin{abstract}
Dose distribution and energy straggling for proton and carbon ion beams in water are investigated by using a hadrontherapy model based on the Geant 4 toolkit. By gridding water phantom in $N \times N \times N$ voxels along $\mathrm{X}, \mathrm{Y}$ and $\mathrm{Z}$ axes, irradiation dose distribution in all the voxels is calculated. Results indicate that carbon ion beams have more advantages than proton beams. Proton beams have bigger width of the Bragg peak and broader lateral dose distribution than carbon ion beams for the same position of Bragg peaks. Carbon ion has a higher local ionization density and produces more secondary electrons than proton, so carbon ion beams can achieve a higher value of relative biological effectiveness.
\end{abstract}

Keywords: Monte Carlo simulation, dose distribution, geant4, Bragg peak.

\section{Introduction}

Early in the forties of last century, Wilson suggested that using the beams of charged particles such as protons and light nuclei for treating malignant tumors [1]. Compared with photons, therapy with protons or heavy ions (such as carbon ions) have advantages in that the beneficial dose profile with a sharp dose fall-off at the end of the particle range [2]. This special advantage makes that killing tumors without destroying healthy tissues becomes possible. Furthermore, an additional advantage of carbon ion irradiation is its increased biological effectiveness in killing tumor cells close to the Bragg peak. Until now proton and ion beams of intermediate energies have become widely used for cancer treatment [3-10]. Many proton therapy facilities and several carbon ion therapy facilities are constructed or are being constructed $[11,12]$. However, carbon ions may have a small amount nuclear fragmentation with medium [13-15]. Doses from secondary nuclear fragments can not only enhance the biological effect, but also have unexpected energy deposition beyond Bragg peak. This feature makes a very selective impact on the tumor possible, and it requires thorough treatment planning based on more reliable calculation. Moreover, long-duration missions on the Earth's moon or exploration of other plants in solar system will be more frequent in the future. One has to pay more attention on space radiation which comprised of high energy protons and high charge and energy nuclei [16]. Further investigations on radiation effects of heavy ions, especially on the spatial effects, are needed to understand the risk of cancer and other diseases from the space radiation. The radiation of ion beams is so essential that the energy deposition and biological effect distribution of ions should be adequately described. In this work, we use the Geant4 toolkit to calculate dose distribution and energy straggling of proton and carbon ion beams in water (a tissue-like medium)

\section{Model and simulation details}

Geant4 is a toolkit for the simulation of the passage of particles through matter. Its areas of application include high energy, nuclear and accelerator physics, as well as studies in medical and space science [17]. In our simulations, the choice of models considers both electromagnetic interactions and hadronic interactions. To ensure the accuracy of our calculations of the depth-dose distribution, the maximum step size in track calculations is taken to be as small as $0.01 \mathrm{~mm}$ during the entire event. In addition, we set a cut value of $0.01 \mathrm{~mm}$ in the whole detector region.

We construct the pure water phantoms with the water density of $1.0 \mathrm{~g} \cdot \mathrm{cm}^{-3}$. During simulation, a typical event is as follows. Firstly, the beam goes through a beam line which includes range shifters, collimators, etc. Then the primary kinematics consists of a single proton or ${ }^{12} \mathrm{C}^{6+}$ particle which hits in a $(40 \mathrm{~mm})^{3}$ water cube perpendicularly. The incident energy for proton is 34 $\mathrm{MeV}, 62 \mathrm{MeV}$, and for carbon ion is $62 \mathrm{MeV} / \mathrm{u}, 113.5$ $\mathrm{MeV} / \mathrm{u}$ respectively. To ensure the accuracy of the calculation of the dose distribution, we run 1,000,000 events for every incident energy.

In order to quantify the amount of energy deposited by ion beams in matter, one usually considers the average energy deposition per unit mass expressed in Gy. These 
characteristics are calculated by gridding a cubic phantom into a matrix of $N \times N \times N$ cubic voxels each with a corresponding lateral dimension and calculating the energy deposited in each of the small cubic voxels. We make a matrix of $200 \times 200 \times 200$ cubic voxels each with a lateral dimension of $0.2 \mathrm{~mm}$. By comparison with the maximum step size $(0.01 \mathrm{~mm})$, the small cubic voxel is big enough to ensure the accuracy of our simulations. Using above small cubic voxels method based on hadrontherapy model, one can get the dose in every small cubic voxel. Moreover, according to the local effect model (LEM) developed in GSI, the value of Relative Biological Effectiveness (RBE) can be calculated for each voxel of the treated volume.

\section{Results and discussion}

Figures 1a-1d show the two dimensional (incident direction and transverse direction) dose distributions for proton and carbon ion beam traversing a water phantom at different energies, respectively. We also give dose contours at the bottom for each case. From Figures 1a and 1c, we can see that they almost have the same position of Bragg peaks (at about $10.5 \mathrm{~mm}$ ) for beams of proton with $34 \mathrm{MeV}$ and carbon ion with $62 \mathrm{MeV} / \mathrm{u}$, so do beams of proton with $62 \mathrm{MeV}$ and carbon ion with 113.5 MeV/u (Bragg peaks at about $29.5 \mathrm{~mm}$ ) in Figures $1 \mathrm{~b}$ and $1 \mathrm{~d}$. This is one reason why we choose these energies for the two kinds of beam, although the main reason is that the two kinds of beam at these energies have been using for hadrontherapy in many facilities [20].
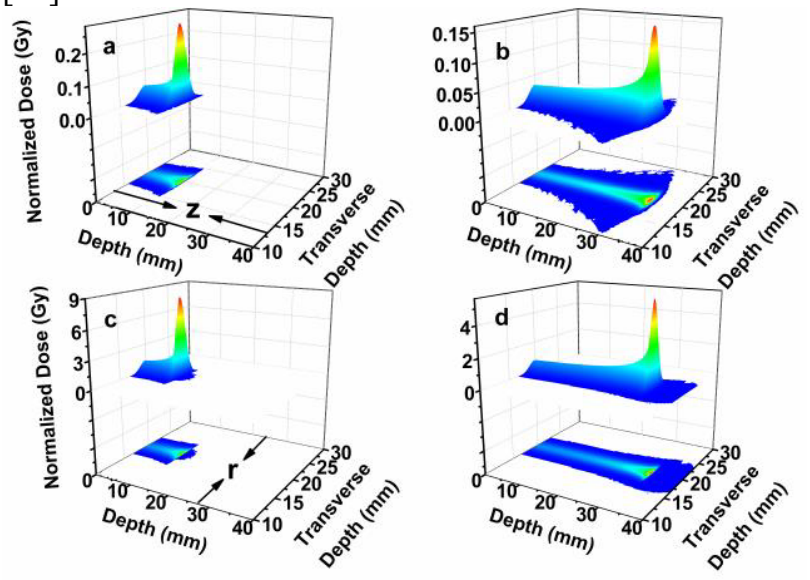

Figure 1. The two dimensional (incident direction and transverse direction) dose distributions in water. Proton beam energy at $34 \mathrm{MeV}$ (a) and $62 \mathrm{MeV}$ (b); Carbon ion beam energy at $62 \mathrm{MeV} / \mathrm{u}$ (c) and $113.5 \mathrm{MeV} / \mathrm{u}(\mathrm{d})$. The normalized doses (Gy) are calculated in $200 \times 200 \times 200$ small cubic voxels, one voxel is $0.2 \times 0.2 \times 0.2(\mathrm{~mm})^{3}$.

The proton beams show more straggling along the incident direction, while the carbon ion beams show distinct tail dose beyond the Bragg peaks. When the carbon ion through water, undergoes a small amount nuclear fragmentation. This process produces a tail of lighter fragments beyond the Bragg peak. The tail reduces the sharp dose contours produced by carbon ion, although for carbon ion it stays within tolerable limits. As shown in Table 1, the dose of Bragg peak is 0.269 Gy for proton beam and 8.56 Gy for carbon ion beam at the same position of Bragg peaks $(10.5 \mathrm{~mm})$. The other group is $0.164 \mathrm{~Gy}$ for proton beam and $5.58 \mathrm{~Gy}$ for carbon ion beam at the same position of Bragg peaks (29.5 mm). The dose of Bragg peak for carbon ion beam is significantly larger than that for proton beam. However, for carbon ion beams, not only the Bragg peaks' width $\left(\sigma_{\mathrm{z}}\right)$ but also lateral width $\left(\sigma_{\mathrm{r}}\right)$ are smaller than that for proton beams when they have the same position of Bragg peak. Figures 2a-2d show the other two dimensional (both transverse directions) dose distributions. We also show dose contours at the bottom for each case. From the figures $2 \mathrm{a}-2 \mathrm{~d}$, except the value of the peaks are very different, the size of dose contours at the bottom for proton beams are slightly bigger than those for carbon ion beams, means that the proton beams have a bigger region of lateral dose distribution as shown in Table 1.

Table 1. Physical quantities of dose distribution for proton and carbon ion beams in water. The position $(R)$, the normalized dose $(D)$, the beam direction width $\left(\sigma_{\mathrm{z}}\right)$, and the lateral width $\left(\sigma_{\mathrm{r}}\right)$ of Bragg peak for $\mathrm{H}^{+}$ions and ${ }^{12} \mathrm{C}^{6+}$ ions at beam energy $(E)$ are given.

\begin{tabular}{|c|c|c|c|c|c|}
\hline Beam & $E(\mathbf{M e V} / \mathbf{u})$ & $R(\mathbf{m m})$ & $\boldsymbol{D}(\mathbf{G y})$ & $\sigma_{\mathbf{z}}(\mathbf{m m})$ & $\sigma_{\mathbf{r}}(\mathbf{m m})$ \\
\hline $\mathrm{H}^{+}$ & 34 & 10.5 & 0.269 & 1.2 & 3 \\
\hline $\mathrm{H}^{+}$ & 62 & 29.5 & 0.164 & 3 & 3 \\
\hline${ }^{12} \mathrm{C}^{6+}$ & 62 & 10.5 & 8.56 & 0.8 & 2.5 \\
\hline${ }^{12} \mathrm{C}^{6+}$ & 113.5 & 29.5 & 5.58 & 1.8 & 2.5 \\
\hline
\end{tabular}
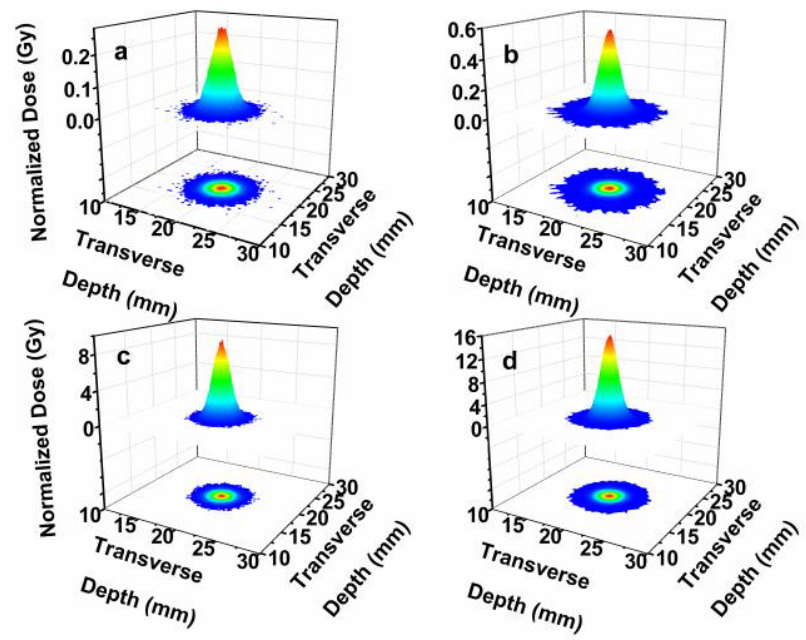

Figure 2. The two dimensional (both transverse directions) dose distributions in water. Proton beam energy at $34 \mathrm{MeV}$ (a) and $62 \mathrm{MeV}$ (b); Carbon ion beam energy at $62 \mathrm{MeV} / \mathrm{u}$ (c) and $113.5 \mathrm{MeV} / \mathrm{u}(\mathrm{d})$. The normalized doses (Gy) are calculated in $200 \times 200 \times 200$ small cubic voxels, one voxel is $0.2 \times 0.2 \times 0.2$ $(\mathrm{mm})^{3}$.

The lateral scattering draws a lot of attentions because it limits the closest approach of a beam passing a critical structure. To give a clear view of dose distribution in detail, we can give any sectional drawing of each dimension. In Figures 3a-3d, we show a sectional drawing of dose distribution which traverses the position 
of center of the transverse depth $(20 \mathrm{~mm})$ for every case, respectively. The value between maximum and minimum has been divided into 20 stripes between color of red to blue. The outermost blue stripe represents the lowest dose which may be mostly formed by the secondary electrons. As we known, in the case of ions inject into a medium, firstly they will have ionization with the atomic shell of the medium's atoms, so they may get electrons, if it happens, the charge of carbon ion has to decrease and the charge of proton may down to zero. Secondly, for carbon ion, it has nuclear reaction processes with the medium and has a lot of secondary fragments such as neutron, proton, alpha and other charged particles, which are responsible for the energy deposition beyond the Bragg peak, they can continue to interact with the medium and produce the electrons, too. However, after the energy straggling, around the Bragg peak, the carbon ion may still has a higher local ionization density than proton and the energies of the secondary electrons are also large. So the blue regions for carbon ion beams are far bigger than those for proton beams, especially beyond the Bragg peaks. Along the beam direction, for proton beams, the green to red stripes appeared in an extended region. It can be said that the carbon ion beams have more steep Bragg peaks than those for proton beams. In Figures $3 \mathrm{e}-3 \mathrm{~h}$, we give a sectional drawing which traverse the position of centre of the Bragg peak for every case, respectively. Except for the outermost blue stripe, we can also find the diameter of the contour for proton beam is about $6.5 \mathrm{~mm}$, but for carbon ion beam, the diameter is only about $5 \mathrm{~mm}$. This means the carbon ion beams have more steep gradients than the proton beams, especially between the red stripe to green stripe.
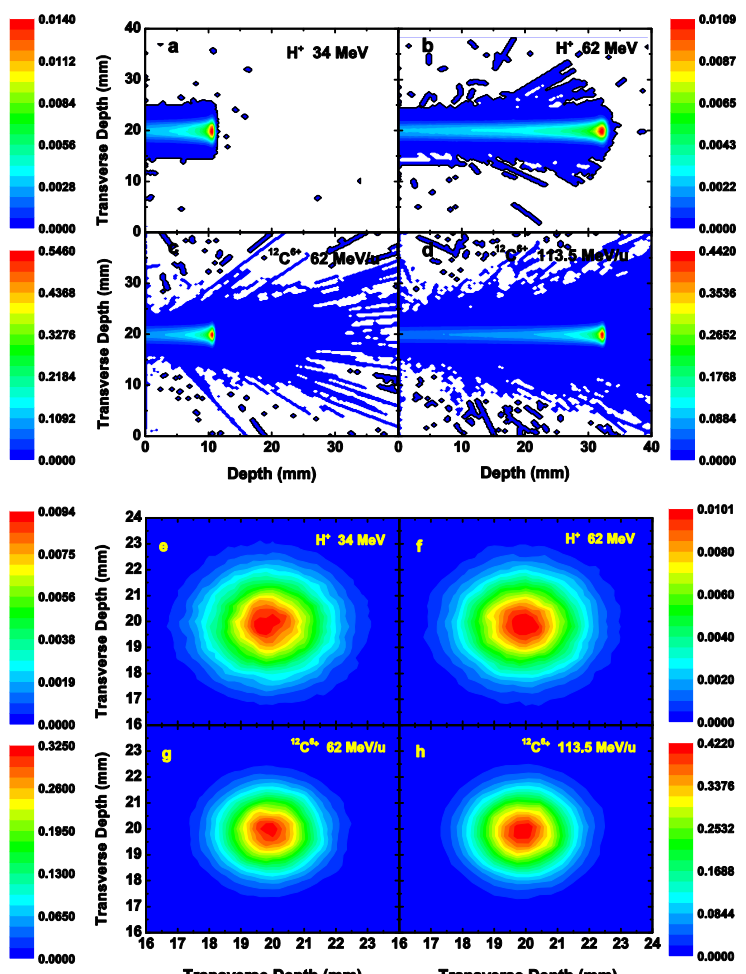

Figure 3. Sectional drawings of dose distributions at the position of center of the transverse depth $(20 \mathrm{~mm})$ along the incident direction. Proton beam energy at $34 \mathrm{MeV}$ (a) and 62
$\mathrm{MeV}$ (b); Carbon ion beam energy at $62 \mathrm{MeV} / \mathrm{u}$ (c) and 113.5 $\mathrm{MeV} / \mathrm{u}$ (d). Sectional drawings of dose distributions at the position of the Bragg peaks along the transverse direction. Proton beam energy at $34 \mathrm{MeV}$ (e) and $62 \mathrm{MeV}$ (f); Carbon ion beam energy at $62 \mathrm{MeV} / \mathrm{u}(\mathrm{g})$ and $113.5 \mathrm{MeV} / \mathrm{u}(\mathrm{h})$. The normalized doses (Gy) are calculated in $200 \times 200 \times 200$ small cubic voxels, one voxel is $0.2 \times 0.2 \times 0.2(\mathrm{~mm})^{3}$.

\section{Conclusion}

In summary, proton beams have broader lateral dose distribution and relatively bigger energy straggling along the beam trajectory than carbon ion beams. For carbon ion, it has a higher local ionization density, so can produce more secondary electrons than proton along the incident direction. All these calculated results and analysis may shed light on application of ion beams for cancer therapy. And this method can also be used to calculate dose distribution in many fields in relation with proton and heavy ion radiations.

\section{Acknowledgments}

This work was supported by the National Natural Science Foundation of China under Grant Nos. 11275071 and 11305061, and the Fundamental Research Funds for the Central Universities under Grant No. 2014MS53.

\section{References}

[1] Wilson R. R., Radiological use of fast protons, Radiology, 47, pp. 487-491, (1946)

[2] Geissel H. \& Scheidenberger C., Slowing down of relativistic heavy ions and new applications, Nucl. Instrum. Meth. B, 114, pp. 136-138, (1998)

[3] Schimmerling W., Vosburgh K. \& Todd P., Interaction of 3.9-Gev nitrogen ions with matter, Science, 174, pp. 1123-1125, (1971)

[4] Schimmerling W., Miller J., Wong M., et al., The fragmentation of $670 \mathrm{~A} \mathrm{MeV}$ neon-20 as a function of depth in water, Radiat. Res., 120, pp. 36-71, (1989)

[5] Xu S., Tay B. K., Tan H. S., et al., Properties of carbon ion deposited tetrahedral amorphous carbon films as a function of ion energy, J. Appl. Phys., 79, pp. 7234-7239, (1996)

[6] Schardt D., Schall I., Geissel H., et al., Charge-changing nuclear reactions of relativistic light-ion beams $(5 \leqslant Z \leqslant 10)$ passing through thick absorbers. Nucl. Instrum. Meth. B, 117, pp. 221-234, (1996)

[7] Amaldi U., Cancer therapy with particle accelerators, Nucl. Phys. A, 654, pp. C375-C399, (1999)

[8] Kraft G., Tumor therapy with heavy charged particles, Prog. Part. Nucl. Phys., 45, pp. S473-S544, (2000)

[9] Matsufuji N., Fukumura A., Komori M., et al., Influence of fragment reaction of relativistic heavy charged particles on heavy-ion radiotherapy, Phys. Med. Biol., 48, pp. 1605-1623, (2003)

[10] Hollmark M., Gudowska I., Belkić, Dž., et al., An analytical model for light ion pencil beam dose 
distributions: multiple scattering of primary and secondary ions, Phys. Med. Biol., 53, pp. 3477-3491, (2008)

[11] Tsujii H., Mizoe J., Kamada T., et al. Overview of clinical experiences on carbon ion radiotherapy at NIRS, Radiother. Oncol., 73, pp. S41-S49, (2004)

[12] Schulz-Ertner D., Nikoghosyan A., Thilmann C., et al., Results of carbon ion radiotherapy in 152 patients, Int. J. Radiat. Oncol. Biol. Phys., 58, pp. 631-640, (2004)

[13] Schardt D., Schall I., Geissel H., et al., Nuclear fragmentation of highenergy heavy-ion beams in water, Adv. Space Res., 17, pp. 87-94, (1996)

[14] Bian B. A., Zhang F. S. \& Zhou H. Y., Fragmentation cross sections of $20 \mathrm{Ne}$ collisions with different targets at $600 \mathrm{MeV} /$ nucleon, Nucl. Phys. A, 807, pp. 71-78, (2008)

[15] Zhao Q., Zhang F. S., Wang Z. P., et al., Secondary Beam Fragments Produced by 200 and $400 \mathrm{MeV} / \mathrm{u}^{12} \mathrm{C}^{6+}$ Ions in Water, Chin. Phys. Lett., 26, pp. 092501, (2009)

[16] Durante M. \& Cucinotta F. A., Heavy ion carcinogenesis and human space exploration, Nature, 8 , pp. 465-472, (2008)

[17] Agostinelli S., Allison J., Amako K., et al., Geant4-a simulation toolkit, Nucl. Instrum. Meth. A, 506, pp. 250-303, (2003)

[18] Scholz M., Kellerer A. M., Kraft-Weyrather W., et al., Computation of cell survival in heavy ion beams for therapy, Radiat. Environ. Biophys., 36, pp. 59-66, (1997) [19] Krämer M., Jäkel O., Haberer T., et al., Treatment planning for heavy-ion radiotherapy: physical beam model and dose optimization, Phys. Med. Biol., 45, pp. 3299-3317, (2000)

[20] Cuttone G., G. Cirrone A. P., Rosa F. Di., et al., Proton therapy detector studies under the experience gained at the CATANA facility, Nucl. Phys. B, 172, pp. 79-83, (2007) 